\title{
CUSP-LIKE FREE-SURFACE FLOWS DUE TO A SUBMERGED SOURCE OR SINK IN THE PRESENCE OF A FLAT OR SLOPHNG BOTTOIVI
}

\author{
G. C. HOCKING ${ }^{1}$
}

(Received 26 September 1983; revised 9 May 1984)

\begin{abstract}
Solutions are found to several problems involving a line source or sink beneath a cusped free surface, over several different impermeable bases. These are compared with known exact and numerical solutions, and with other work, both theoretical and experimental, on similar problems.
\end{abstract}

\section{Introduction}

Using conformal mapping techniques, Tuck and Vanden Broeck [8], subsequently referred to as $I$, found a series solution to the problem of a line source or sink situated beneath a free surface in a two-dimensional, steady, irrotational flow of an incompressible, inviscid, infinitely deep fluid under gravity. They found that a cusp-shaped free surface existed only for a single Froude number, namely 1.776.

To understand why flows of this type exist only at a single Froude number, we must look at a slightly more general problem. If a sink is placed beneath the interface between two fluids of different density, with the lighter fluid above, then for any flow with a Froude number greater than some critical value, both fluids will be drawn into the sink. In this situation, the angle of entry of the interface into the sink will lie somewhere between zero and $\pi / 2$, and will tend toward the latter as the Froude number tends to the critical value from above. Huber [5] calculated several such flows in the case where the line sink is "located in the bottom corner of a rectangular configuration with the upstream end extending to infinity."

\footnotetext{
${ }^{1}$ Applied Mathematics Department, University of Adelaide, Adelaide, S.A. 5000.

(C) Copyright Australian Mathematical Society 1985, Serial-fee code 0334-2700/85
} 
At and below this critical value of Froude number, the lighter fluid will remain motionless, and the condition of constant pressure across the interface will reduce to a condition of constant pressure on a free surface. The behaviour of the free surface at these lower Froude numbers is unclear. Several earlier researchers [6], [10] considered flows for small values of Froude number, where a stagnation point exists above the source on the free surface. Short waves appear in these stagnation-point flows, and these steepen as the Froude number increases. Solutions of this type appear to exist only for values of Froude number less than unity, leaving a region in the Froude number space about which little is known.

The cusp-like flows in which we are interested are the flows at the critical Froude number, where the lighter fluid begins or ceases to flow.

Craya [2] (also in Yih [13], pages 124-126 and Tuck [9]) used an inverse procedure first derived by Sautreaux [7], to find an exact solution to a similar problem in which the source or sink is located on the top of an infinite triangular "mountain" of apex angle $120^{\circ}$, beneath the free surface. Using an approximate and intuitive argument, Craya produced an estimate for the solution to the problem considered in $I$. The same argument can be used to make estimates for the Froude number at which cusp-like solutions occur in the more general case when the apex angle of the "mountain" lies between $0^{\circ}$ (infinite depth) and $180^{\circ}$ (rigid horizontal plane). The right half of this flow is depicted in Figure 1.

Here we consider this problem using a numerical method based upon that used in $I$. If we let the "mountain" apex angle be $2 \pi \gamma$, then there are three cases of particular interest. The problem in $I$ occurs when $\gamma=0$, while for $\gamma=\frac{1}{2}$ the source is located on a rigid horizontal plane. The case $\gamma=\frac{1}{3}$, i.e. for an apex angle of $120^{\circ}$, is the same problem for which Craya found an exact solution, and we can use it as an accuracy test for the numerical method.

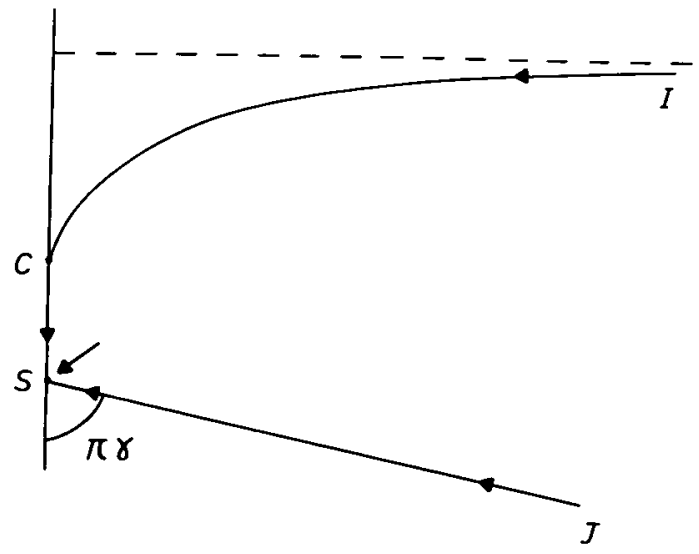

Figure 1. The physical z-plane. 
In Section 4, we look at a related problem, where the source is situated above a horizontal plane, and once again seek solutions with a cusped free surface. Gariél [3], performed an experiment with a flow of this type to approximate the case $\gamma=0$, i.e. infinite depth, and test the prediction of Craya.

Note that although the mathematics is done for a source, the more applicable case of a sink may be recovered by letting the source strength be negative.

\section{An exact solution}

In [9], Tuck derived an inverse procedure for generating solutions to free-surface problems. The procedure is equivalent to those derived by Sautreaux [7] and others (see Wehausen and Laitone [12], page 736).

The equation

$$
\frac{d z}{d f}=i Y^{\prime}(f)+\left[\frac{-1}{2 g Y(f)}-\left(Y^{\prime}(f)\right)^{2}\right]^{1 / 2}
$$

for a suitable choice of $Y(f)$, defines an exact flow with a free surface $z(\phi, 0)$, where $f(z)=\phi(x, y)+i \psi(x, y)$ is a complex velocity potential.

Our task in this section is to examine the equations which result if we make the substitution

$$
Y(f)=-a \exp (-\pi f / m)
$$

in (1), and discover what type of fluid flow they describe. We will see that the flow is like that described in the introduction, where a source is located on a triangular "mountain" with an apex angle of $120^{\circ}$ i.e. $\gamma=\frac{1}{3}$. Since the method is an inverse procedure, there is no systematic way to find a substitution $Y(f)$ which will provide an exact solution for other values of $\gamma$, and hence such exact solutions are unavailable to us.

Substituting (2) into (1) gives

$$
z(f)=-a \beta Z\left(-\frac{\pi f}{m}-\log \beta\right)
$$

where

$$
\beta=\left(\frac{m^{2}}{2 g a^{3} \pi^{2}}\right)^{1 / 3}
$$

and

$$
Z^{\prime}(G)=i e^{G}+e^{G}\left(-1+e^{-3 G}\right)^{1 / 2}
$$

where $g$ is the acceleration due to gravity. 
The equations (3)-(5) are a little difficult to interpret, but we can transform them to a more easily studied form. If we let

$$
f_{T}=f+\frac{m}{\pi} \log \beta=\frac{m}{3 \pi} \log \left(4 t(t+1)^{-2}\right)
$$

and substitute into equations (3)-(5), then nondimensionalize with respect to the length $\left(m^{2} / 18 \pi^{2} g\right)^{1 / 3}$ and the velocity $(2 m g / 3 \pi)^{1 / 3}$ we acquire two new equations in the nondimensional variables $f_{N}$ and $z_{N}$ i.e.

$$
f_{N}(t)=\log \left(4 t(t+1)^{-2}\right)
$$

and

$$
\frac{d z_{N}}{d t}=-i\left(\frac{2}{3}\right)^{1 / 3} t^{-1 / 3}(t+1)^{-4 / 3}(t-1)
$$

which satisfy the nondimensional free-surface condition

$$
y_{N}+\left|f_{N^{\prime}}\left(z_{N}\right)\right|^{2}=0
$$

on $\psi_{N}=0, \phi>0$.

It is convenient at this point to discard the subscript ' $N$ ' which denotes a nondimensional variable, and henceforth assume all variables are in nondimensional co-ordinates unless otherwise stated.

To ascertain the character of this inverse solution we must determine the nature of the singularities at $t=0$ and $t=-1$, and the boundaries of the flow domain.

As $t$ tends toward zero, $f \rightarrow \log t$ and $z+i \bar{h}_{s} \rightarrow$ const $\cdot t^{2 / 3}$ where $-i \bar{h}_{s}$ is the value of $z$ at $t=0$, so that

$$
f \rightarrow \frac{3}{2} \log \left(z+i \bar{h}_{s}\right)
$$

and hence the flow is like a source of strength $3 \pi$ located at $z=-i \bar{h}_{s}$. Near the point $t=-1, f \rightarrow-2 \log (t+1)$ and $z \rightarrow$ const. $(t+1)^{-1 / 3}$, and therefore

$$
f \rightarrow 6 \log z \text {. }
$$

The transformation (7) maps $f=\phi+i \psi$ to the lower half of the unit circle. On the real $t$-axis, $\psi$ takes the value of $-\pi$ for $-1<t<0$, and zero for $0<t \leqslant 1$. $\psi$ is also zero on the lower semi-circumference of the unit circle, the line which corresponds to the free surface. Observe that $\psi$ only changes value at the singular points $t=0$ and $t=-1$, which means that the lines which map from the $z$-plane to the boundary of the lower half of the unit circle in the $t$-plane are stream surfaces, and hence possible solid boundaries of the region of flow. (See Figures 2 and 3.)

In a fashion very similar to a Schwarz-Christoffel conformal mapping, the exact solution relates the $z$-plane to the $t$-plane by a differential expression. The nature of the singularities at $t=0$ and $t=-1$ indicate that the $z$-plane is as for the case $\gamma=\frac{1}{3}$ discussed in the introduction, and shown in Figure 1 . 


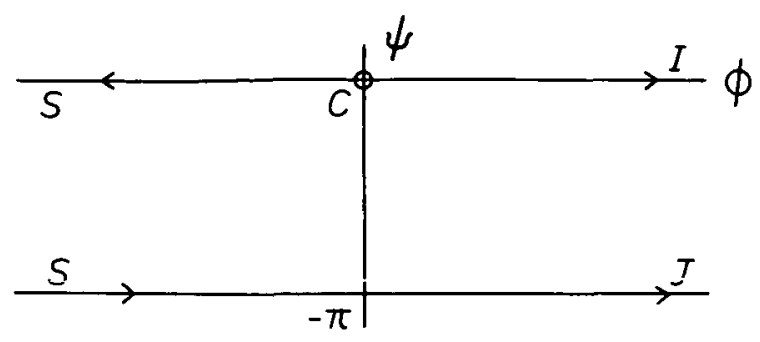

FIGURE 2. The complex velocity potential $f$-plane.

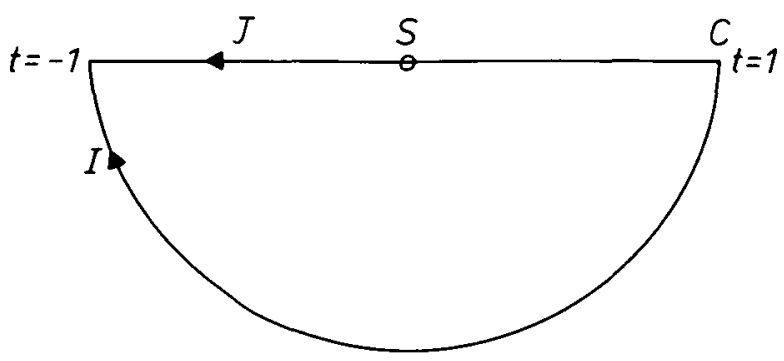

Figure 3. The $t$-plane.

The quantity $m$ which appears in (2), can be seen from the length and velocity scales to have the dimensions of a flux, and is in fact the dimensional strength of the source per unit length.

The velocity field of the flow can be written

$$
q^{*}=\frac{d f}{d z}=-i\left(\frac{3}{2}\right)^{1 / 3} t^{-2 / 3}(t+1)^{1 / 3}
$$

and since the cusp is located at $t=1$, it is easily seen from the free-surface condition (9) that the nondimensional depth $\bar{h}_{c}$ of the cusp is

$$
-\left|f^{\prime}(z)\right|_{t=1}^{2}=-\bar{h}_{c}=-3^{2 / 3} \text {. }
$$

Hence

$$
z(t)=\int_{1}^{t}-i\left(\frac{2}{3}\right)^{1 / 3} \tau^{-1 / 3}(\tau+1)^{-4 / 3}(\tau-1) d \tau-i 3^{2 / 3}
$$

The source is located at $t=0$, and it is possible, by integrating (11), to show that the nondimensional depth of the source in the $z$-plane, $\bar{h}_{s}$, is

$$
\bar{h}_{s}=\frac{1}{2} 3^{2 / 3} B\left(\frac{2}{3}, \frac{1}{2}\right)
$$

where $B$ denotes the Beta function [1].

To define the Froude number, let us return to the problem for general $\gamma$ for a moment. The free-surface condition (9) for the general problem is a result of 
nondimensionalization with respect to the length $\left(m^{2}(1-\gamma)^{2} /\left(8 \pi^{2} g\right)\right)^{1 / 3}$ and the velocity $(m g(1-\gamma) / \pi)^{1 / 3}$. We define the Froude number with respect to the amount of fluid which flows into the flow domain. The flux from the source per unit length is $m$, but fluid only flows out of the source from the region of interest through an arc of $\pi(1-\gamma)$ radians. Thus the flux is $(m / 2)(1-\gamma)$ into the right half of the flow domain.

If we define the Froude number in terms of this quantity and the (dimensional) depth of the source $h_{s}$, i.e.

$$
F=\left(\frac{m^{2}(1-\gamma)^{2}}{4 g h_{s}^{3}}\right)^{1 / 2},
$$

then in terms of the nondimensional source depth $\bar{h}_{s}$, the Froude number is

$$
F=\left(\frac{2 \pi^{2}}{\bar{h}_{s}^{3}}\right)^{1 / 2}
$$

Thus the Froude number corresponding to the exact solution i.e. for the case $\gamma=\frac{1}{3}$, is

$$
F=\left(\frac{16 \pi^{2}}{9 B\left(\frac{2}{3}, \frac{1}{3}\right)}\right) \doteq 1.006 .
$$

Craya assumed that only the flux from the source would alter if the angle $\gamma$ were changed, so that he predicted

$$
F_{c r}=3(1.006)(1-\gamma) / 2 \text {. }
$$

This assumption, while it does give a rough approximation to the actual value of $F$ for each $\gamma$, is later shown to be incorrect.

\section{Solution for arbitrary apex angle}

In this section we develop a numerical scheme, based upon that in $I$, to calculate solutions to the problem for values of $\gamma$ between zero and one half.

To begin, it is necessary to reintroduce the complex variable $t$, related by (7) to $f$. The relationship between $z$ and $t$ is chosen so that $z$ is a power series in $t$ of the form

$$
z(t)=-i X-i t^{-\gamma}\left(\gamma-\frac{1}{2}\right)^{-1}\left((t+1)^{2 \gamma-1}-1\right) \sum_{j=0}^{\infty} a_{j} t^{j}
$$

where the constant $X$ and all of the coefficients $a_{j}, j=0,1,2, \ldots$, are real. Note that the source depth $\bar{h}_{s}$, i.e. the depth of the top of the "mountain", is equal to the value of $X$. 


\begin{tabular}{|c|c|r|}
\hline$j$ & \multicolumn{2}{|c|}{$a_{j}$} \\
\hline & Numerical & \multicolumn{1}{c|}{ Exact } \\
\hline 0 & -0.6555 & -0.6552 \\
1 & 0.1748 & 0.1747 \\
2 & -0.0169 & -0.0170 \\
3 & 0.0061 & 0.0062 \\
4 & -0.0031 & -0.0033 \\
5 & 0.0019 & 0.0020 \\
6 & -0.0013 & -0.0014 \\
\hline
\end{tabular}

TABLE 1. Comparison of the numerically calculated coefficients $a_{j}$ for $N=55$, with those calculated from the exact solution at $\gamma=\frac{1}{3}$.

Representing $z(t)$ in this form rather than as a derivative, as in equation (8), removes the necessity for numerical integration to find the value of $z$. The disadvantage of this form is that since (8) cannot be integrated in closed form, (13) does not reduce to a simple expression when $\gamma=\frac{1}{3}$. It is possible, however, to calculate all of the $a_{j}$ for the case $\gamma=\frac{1}{3}$, by differentiating (13) and equating the coefficients of $t^{j}$ with those in (8). The first seven coefficients calculated in this manner are shown in Table 1.

As we have seen (7) ensures that the boundaries of the lower half of the unit circle in the $t$-plane are stream surfaces. Since $X$ and the $a_{j}$ are real, (13) maps the line $x=0$ in the $z=x+i y$ plane, to $0<t \leqslant 1$, and the right hand side of the sloping wall to $-1<t<0$. It is possible to consider $x=0$ as a solid boundary because it is a line of symmetry of the flow. The left hand side of the flow maps to the upper half of the unit circle in the $t$-plane.

Thus all of the conditions on the flow are satisfied by (7) and (13) except that of constant pressure on the free surface. In the $t$-plane this condition (9) can be written as

$$
y(t)+\left|f^{\prime}(t)\right|^{2}\left|z^{\prime}(t)\right|^{-2}=0
$$

on $t=e^{-i \theta}, 0 \leqslant \theta \leqslant \pi$.

Substituting (7) and (13) into (14) gives a set of equations for the unknown coefficients $a_{j}$ of the series. Also, since we are searching for cusp-like solutions only, we specify

$$
\frac{d z}{d t}=0 \quad \text { at } t=1
$$

which gives an extra condition on the unknowns, namely

$$
\sum_{j=0}^{\infty} a_{j}\left((j-\gamma)\left(2^{2 \gamma-1}-1\right)+(2 \gamma-1) 2^{2 \gamma-2}\right)=0 .
$$


The full problem then is to find $X$ and all of the $a_{j}, j=0,1,2, \ldots$, such that $P\left(\theta ; a_{j}\right)=0$ for $0 \leqslant \theta \leqslant \pi$, subject to (16), where

$$
P\left(\theta ; a_{j}\right)=Y(\theta)+4\left(\gamma-\frac{1}{2}\right)^{2} r^{-4 \gamma} \sin ^{2} \theta\left[A^{2}(\theta)+B^{2}(\theta)\right]^{-1}
$$

and

$$
\begin{array}{r}
Y(\theta)=-X-\left(\gamma-\frac{1}{2}\right)^{-1} \sum_{j=0}^{\infty} a_{j}\left(r^{2 \gamma-1} \cos \left(j-\frac{1}{2}\right) \theta-\cos (\gamma-j) \theta\right) \\
\begin{array}{r}
A(\theta)=\sum_{j=0}^{\infty} a_{j}\left[(j-\gamma)\left(r \cos \left(j+\gamma-\frac{1}{2}\right) \theta-r^{2-2 \gamma} \cos j \theta\right)\right. \\
+(2 \gamma-1) \cos (j+\gamma) \theta], \\
B(\theta)=\sum_{j=0}^{\infty} a_{j}\left[(j-\gamma)\left(-r \sin \left(j+\gamma-\frac{1}{2}\right) \theta+r^{2-2 \gamma} \sin j \theta\right)\right. \\
-(2 \gamma-1) \sin (j+\gamma) \theta]
\end{array}
\end{array}
$$

and

$$
r=2 \cos (\theta / 2), \quad 0 \leqslant \theta \leqslant \pi .
$$

We cannot find an analytic solution to this problem, but if we truncate the series to $N$ terms, and consider $M$ values of $\theta$, we have a system of $M$ equations, plus the cusp condition (16), for the unknown coefficients. The system is nonlinear, but we can solve it using Newtonian iteration.

Before proceeding to look at the details of the numerical procedure, we should consider the special case where the source is located on a horizontal plane, i.e. $\gamma=\frac{1}{2}$, since, unlike the cases for $\gamma<\frac{1}{2}$, the fluid at infinity is no longer a stationary fluid, but a uniform flowing stream. The limiting case of (13) as $\gamma \rightarrow \frac{1}{2}$ takes this fact into account, since as $\gamma \rightarrow \frac{1}{2}$

$$
\left((t+1)^{2 \gamma-1}-1\right)\left(\gamma-\frac{1}{2}\right)^{-1} \rightarrow 2 \log (t+1)
$$

and then as $t \rightarrow-1$

$$
f \rightarrow-2 \log (t+1)
$$

and

$$
z \rightarrow-2 \sum_{j=0}^{\infty} a_{j}(-1)^{j} \log (t+1)
$$

so that

$$
f \rightarrow \bar{U}_{\infty} z
$$

where

$$
\bar{U}_{\infty}=\left(\sum_{j=0}^{\infty} a_{j}(-1)^{j}\right)^{-1}
$$

is the stream velocity at infinity. 


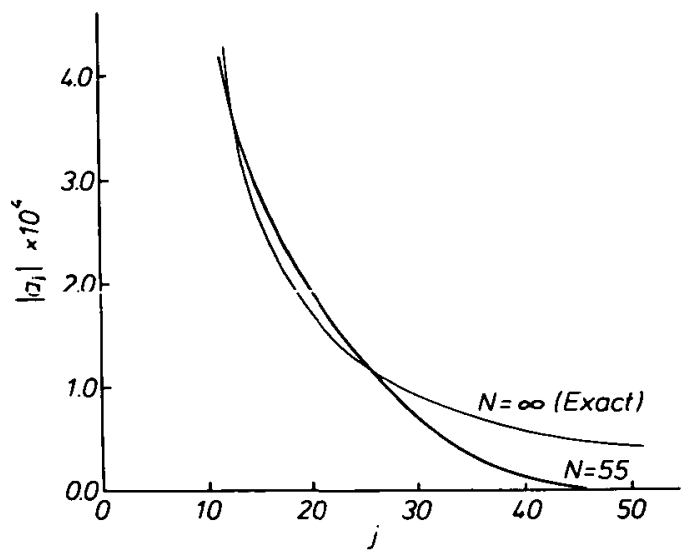

FIgURE 4. Comparison of the rate of decay of the coefficients $a_{j}$ for increasing $j$ calculated numerically with $N=55$, and those calculated from the exact solution, at $\gamma=\frac{1}{3}$.

To obtain solutions, at any value of $\gamma$, we choose a set of $M$ equally spaced, discrete values of $\theta_{k}$, and iterate until $P\left(\theta_{k} ; a_{j}\right)$ is less than some small number $\left(10^{-7}\right.$ say) at all values of $\theta_{k}$. We solve the equations for some small value of $N(5$ say), and then use this set of coefficients as a starting guess for an increased value of $N$.

The choice of $M=N-1$ allows us to have $X$ (and hence the Froude number) as an input parameter. We would expect that no solution could be found for general $\gamma$ and $X$ since we expect steady solutions with a cusp to exist only at a single Froude number for a given value of $\gamma$. Several attempts at various values of $\gamma$ and $X$ failed to show any sign of convergence of the iterative scheme, reinforcing this idea.

On the other hand, the choice $M=N$, which allows $X$ to be a variable of the numerical problem, resulted in quite rapid convergence of the scheme for almost all values of $\gamma$, with less than four iterations required at each value of $N$. The values of $\gamma$ for which convergence did not occur were those very close to $\gamma=\frac{1}{2}$, where the $\gamma-\frac{1}{2}$ term in the denominator of $z(t)$ becomes very small. This difficulty was overcome in the case $\gamma=\frac{1}{2}$ by using the mapping

$$
z(t)=-i X-2 i t^{-1 / 2} \log (t+1) \sum_{j=0}^{\infty} a_{j} t^{j}
$$

and substituting as before.

Calculations were found to be accurate to 3 figures for all values of $\gamma$ when we chose $N=55$. In the case where $\gamma=0$, as in $I$, the solution converged to 5 figure accuracy when only 30 coefficients of the series had been calculated.

The behaviour of the numerical calculations was similar for all values of $\gamma$ such that $0<\gamma<\frac{1}{2}$, and hence we may use the "exact" series solution at $\gamma=\frac{1}{3}$ to 


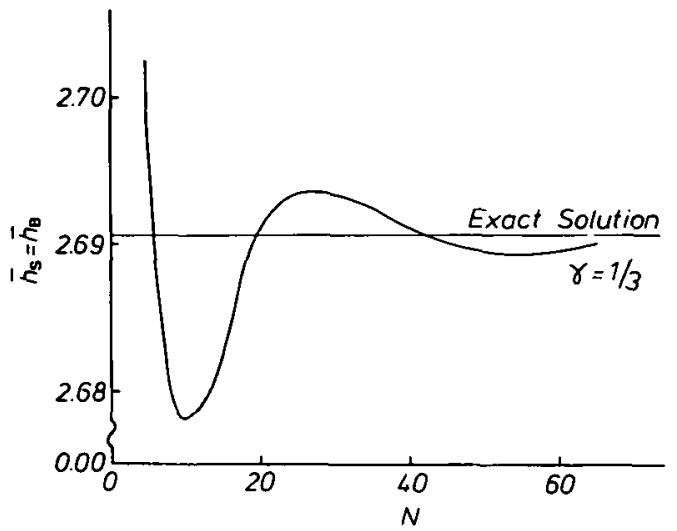

FIgURE 5. Convergence of the numerical method to the exact solution for the case $\gamma=\frac{1}{3}$, as $N$ is increased.

analyse our results. A comparison of the numerically and exactly calculated coefficients for $\gamma=\frac{1}{3}$ is given in Table 1 and Figure 4. The graphical representation shows clearly how the truncation of the series to a finite number of terms affects the results.

A Domb-Sykes plot [4] (see also [11]) of the exactly calculated series for $\gamma=\frac{1}{3}$, indicates that there is a branch-point singularity at $t=-1$, which means the radius of convergence of the series is unity. It is this fact which accounts for the relatively slow decay of the coefficients $a_{j}$ for large $j$. In fact, for large $j, a_{j} \sim$ $0.006\left(\frac{1}{\frac{1}{j}}\right)$.

The highly converged series solution, for the case $\gamma=0$, has a Domb-Sykes plot which gives the nearest singularity to be near the point $t=-1.4$. The radius of convergence of this series is greater than unity, and hence the coefficients decay quickly to zero. This different behaviour at $\gamma=0$ suggests that perhaps the inclusion of a sloping wall in the problem introduces a branch-point singularity at

\begin{tabular}{|c|c|}
\hline$N$ & Source Depth \\
\hline 10 & 2.6782 \\
20 & 2.6913 \\
30 & 2.6934 \\
40 & 2.6910 \\
50 & 2.6894 \\
60 & 2.6896 \\
65 & 2.6901 \\
$\infty$ (exact) & 2.6907 \\
\hline
\end{tabular}

TABLE 2. Table showing the convergence of source depth toward the exact solution for increasing values of $N$, for the case $\gamma=\frac{1}{3}$. 


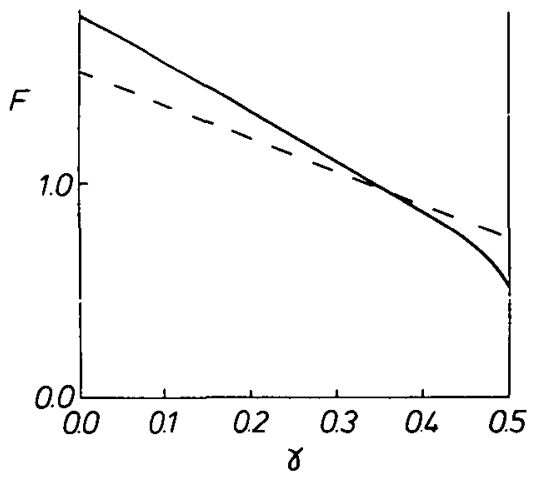

Figure 6. Comparison of Craya's results..., with ours-, for changing values of $\gamma$. Depths are measured from the stagnation level.

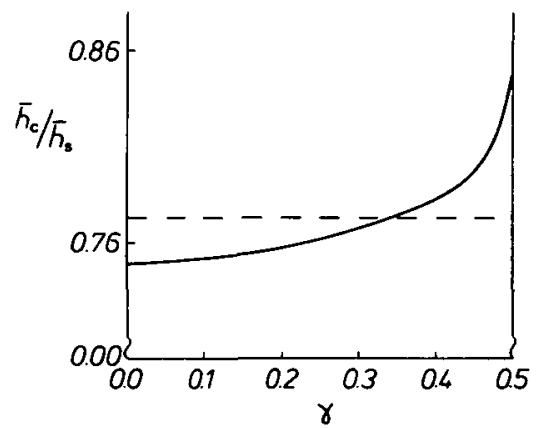

FIGURE 7. Ratio of cusp depth to source depth plotted against $\gamma$, compared with Craya's estimate.

$t=-1$. Figure 5 shows graphically the oscillatory nature of the convergence of the numerical scheme toward the exact solution for $\gamma=\frac{1}{3}$. (See also Table 2.)

Bearing these facts in mind, we can now move to a discussion of the results for general values of $\gamma$. The Froude number at which the cusp-like solutions occur was found to vary with $\gamma$ (Figure 6), and the variation of $F$ and the source and cusp depths with $\gamma$ is shown in Table 3. The error in the assumption of Craya is perhaps most clearly illustrated in Figure 7, which shows the ratio of cusp depth to source depth i.e. $\bar{h}_{c} / \bar{h}_{s}$ plotted against $\gamma$. Craya's assumption implies that this

\begin{tabular}{|c|c|c|c|}
\hline Angle $\gamma$ & Cusp Depth & Source Depth & $F$ \\
\hline 0.0 & 1.3808 & 1.8426 & 1.7763 \\
0.25 & 1.8256 & 2.3915 & 1.2013 \\
0.33 & 2.0785 & 2.6894 & 1.0074 \\
0.45 & 2.6586 & 3.3377 & 0.7286 \\
0.50 & 3.6879 & 4.3163 & 0.4955 \\
\hline
\end{tabular}

TABLE 3. Results for various values of bottom slope $\gamma$, computed with 50 coefficients in the series. Depths are measured from the stagnation level. 
ratio is constant for all values of $\gamma$, but the results using the method in this paper, clearly show an increase in this ratio as $\gamma$ increases.

The definition of $F$ for all values of $\gamma$ with $0 \leqslant \gamma<\frac{1}{2}$ is based on the depth of the source beneath the level of the fluid at infinity, which is the stagnation level. For consistency, the stagnation level was also used as a base for depth measurements for the case $\gamma=\frac{1}{2}$, but, as mentioned previously, the fluid in this situation does not have a stagnation region at infinity. A more useful definition of the Froude number at $\gamma=\frac{1}{2}$ would be one based upon a length scale taken as the depth of the source beneath the level of the free surface at infinity, where the fluid is flowing with a constant velocity $\bar{U}_{\infty}$. The resulting value of the modified Froude number is $F=1.44$. Free-surface profiles for several values of $\gamma$ are shown in Figures 8(a), (b).

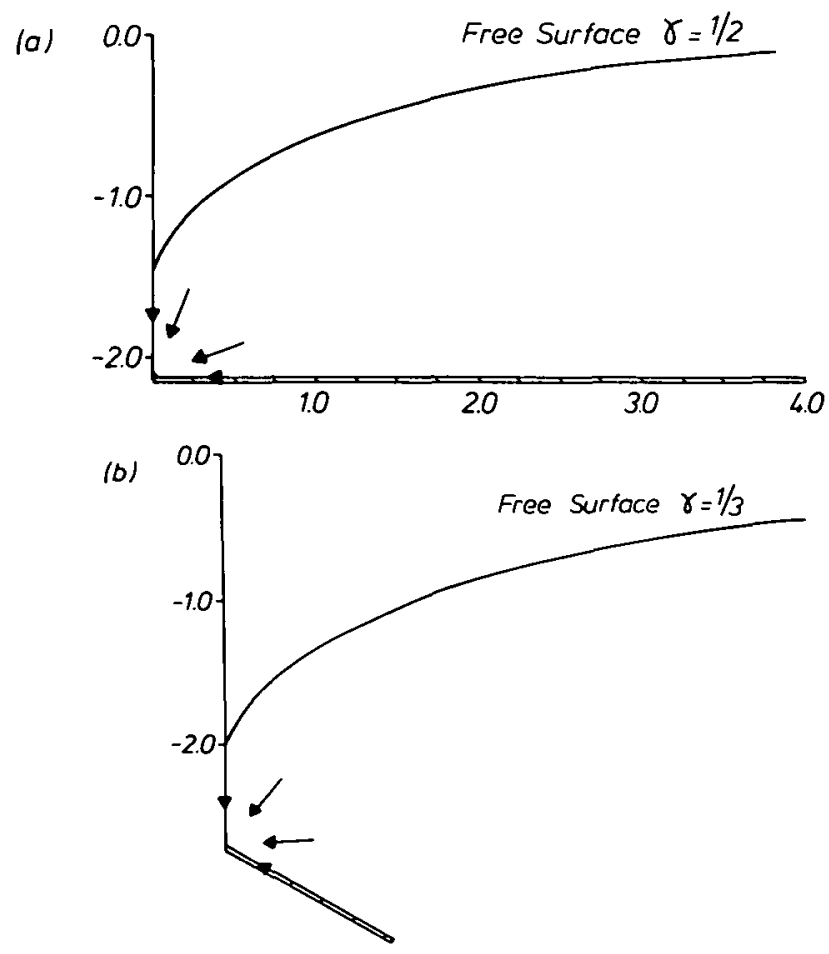

FIGURE 8. Free-surface profiles for the source on (a) a rigid horizontal plane, (b) the top of a mountain with an apex angle of $120^{\circ}$.

\section{A source above a horizontal plane}

In this section we consider a problem similar in nature to that discussed earlier, but with a different wall geometry. Let the source now be situated at a finite distance above an impermeable horizontal plane of infinite extent, as in Figure 9. 


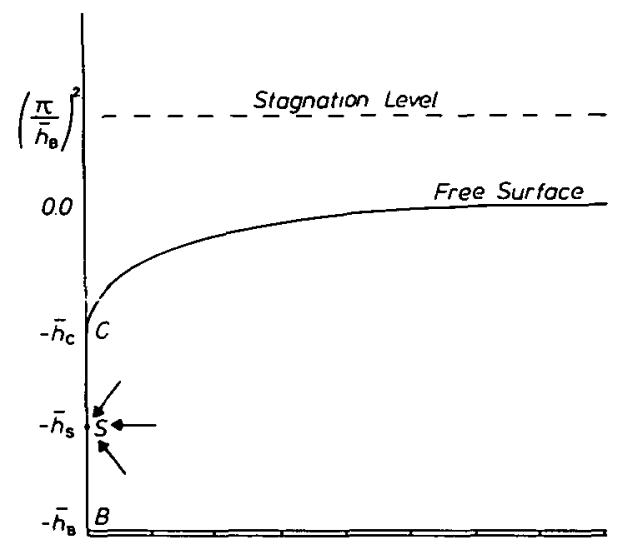

FIGURE 9. Physical z-plane for a source above a horizontal plane.

We have calculated two limiting cases of this situation already; the case where the bottom depth tends to infinity is as considered in $I$, and the situation where the source lies on the horizontal plane is precisely the case $\gamma=\frac{1}{2}$ in the previous section of the present paper. Note that in this section, all of the depths are to be measured from the level of the fluid at infinity, which is no longer the stagnation level since the flow tends toward a uniform stream at large distances from the source. All distances measured from the stagnation level will be denoted $h^{*}$.

Once again we choose a series form for $z(t)$ such that (7) ensures the boundaries are stream surfaces, and then find a set of coefficients which satisfy the free-surface condition. Without loss of generality we can change the constant in Bernoulli's equation and thus replace ( 9 ) by

$$
y+\left|f^{\prime}(z)\right|^{2}=\left(\pi / \bar{h}_{B}\right)^{2}
$$

where $\bar{h}_{B}$ is the depth of the plane, and therefore $\pi / \bar{h}_{B}=\bar{U}_{\infty}$, the velocity of the uniform stream. Thus at large distances from the source $\left|f^{\prime}(z)\right| \rightarrow \bar{U}_{\infty}$ and hence $y \rightarrow 0$ on the free surface. A suitable transformation for $z(t)$ is

$$
z(t)=-i K-2 i(t+c)^{1 / 2} \log (t+1) \sum_{j=0}^{\infty} a_{j} t^{j-1}
$$

for some constant $c, 0 \leqslant c \leqslant 1$. Note that points $-c<t<0$ map to $x=0$ beneath the source, $t=-c$ maps to the stagnation point on the plane beneath the source, and $-1<t<-c$ to points along the impermeable horizontal plane.

The geometry of the problem allows us to define the Froude number in two ways, both of which are consistent with the definition already given. In fact, both are useful parameters in analysing our results. We allow $F$ to remain the Froude number based on the depth of the source $h_{s}$, and define a new parameter $F_{0}$, 
which we base on the depth of the plane $h_{B}$, so that

$$
F_{0}=\left(\frac{m^{2}(1-\gamma)^{2}}{4 g h_{B}^{3}}\right)^{1 / 2}=\frac{U_{\infty}}{\sqrt{g h_{B}}}=\left(\frac{2 \pi^{2}}{\bar{h}_{B}^{3}}\right)^{1 / 2} .
$$

Note that this latter definition is the conventional one in hydraulics, founded on free-stream velocity and fluid depth. Flows with $F_{0} \lessgtr 1$ can be described as super- and sub-critical respectively.

The numerical procedure itself is very similar to that in Section 3. A set of nonlinear algebraic equations is formed by substituting (7) and (20) into (19) on $t=e^{-i \theta}, 0<\theta<\pi$, and truncating the series in (20) to a finite number of terms. We then force the equation to hold at $N$ distinct, equally spaced values of $\theta$. We once again use (15) to force the free surface to have a cusp shape, giving rise to another equation.

Since only one Froude number will give rise to a cusp-like flow for each geometrical configuration, we let $c$ be an input parameter and $K=\bar{h}_{B}$, and $\bar{h}_{s}$ be output parameters.

The number of iterations required at each value of $N$ is similar to the number required in the situation with a sloping bottom, but the accuracy is only two-figure at $N=55$, as can be seen in Table 4, which shows typical convergence behaviour of the source depth $\bar{h}_{s}$ as $N$ increases, for nonzero values of $c$.

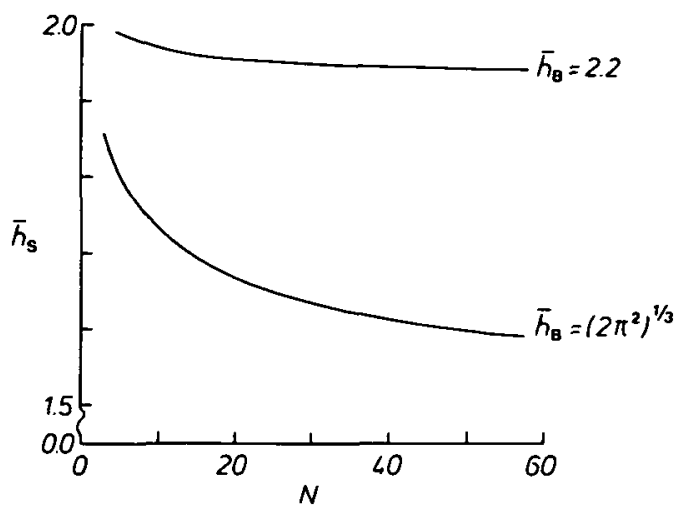

Figure 10. Convergence for increasing $N$ for two cases with the source above the horizontal plane.

\begin{tabular}{|c|c|}
\hline$N$ & $\bar{h}_{s}$ \\
\hline 25 & 1.9538 \\
35 & 1.9481 \\
45 & 1.9441 \\
55 & 1.9411 \\
\hline
\end{tabular}

TABLE 4. Convergence of the source depth $\bar{h}_{s}$ for a typical case where the source is situated above a horizontal plane $\left(c=0.045, \bar{h}_{B} \doteq 2.2\right)$ for increasing values of $N$. 
Note that the convergence is monotonic, in contrast to the oscillatory convergence seen in Section 3 (see Figures 5 and 10). The series coefficients for the two situations behave in a very similar manner to each other; i.e. both have alternating signs and a similar rate of decay of the coefficients $a_{j}$ to zero for increasing $j$. The fact that the case $c=0$, where the source lies on the horizontal plane, behaves like the sloping base problems in this regard, indicates that the sudden change in the nature of the convergence may be caused by the introuluction of a stagnation point on the plane beneath the source, i.e. at $z=-i \bar{h}_{B}$.

Calculations proceeded quite smoothly for values of $c$ between zero and 0.3 . For values of $c$ greater than 0.3 , the numerical scheme began to converge much more slowly toward a solution, making the results unreliable, and if the program was run with $c$ much greater than 0.35 , no convergence at all was apparent.

It appears to be more than coincidence that the value of $c=0.3$ corresponds closely to a value of base depth $K_{c r}=\bar{h}_{B}=\left(2 \pi^{2}\right)^{1 / 3}$, which gives a Froude number of $F_{0}=1$. This means the numerical method breaks down in the transition region between super- and sub-critical flows. This would suggest that perhaps the method used in this paper will not handle the type of cusp-like flow which exists in the region $0<F_{0}<1$, if solutions do exist.

At $F_{0}=0$, where the source is an infinite distance above the horizontal plane, as in $I$, a highly accurate solution is available. One possibility might be that for subcritical flow, $F_{0}<1$, waves form on the free surface at infinity which have decreasing amplitude for decreasing $F_{0}$, and which disappear completely at $F_{0}=0$.

Within the range of values of $K$ for which our solutions are reliable i.e. $2.120 \leqslant K \leqslant K_{c r}=\left(2 \pi^{2}\right)^{1 / 3}$ there are several interesting features.

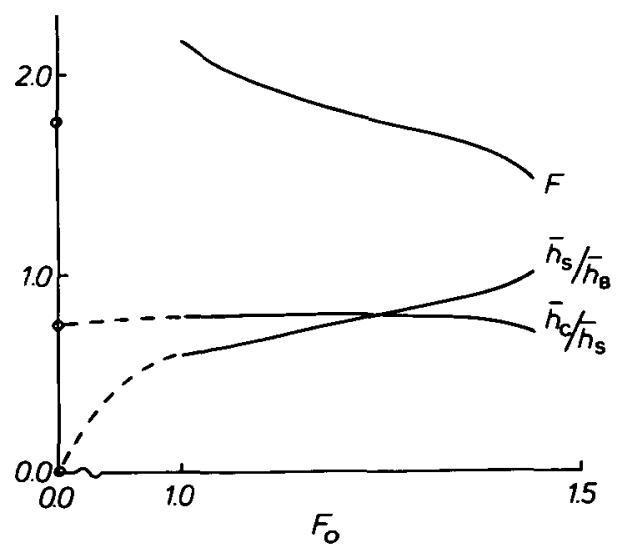

FIGURE 11. Results for the source above the plane where $1 \leqslant F_{0} \leqslant 1.44$, showing also the values for the infinite depth case i.e. $F_{0}=0$. 
Firstly, the depth of the horizontal plane beneath the stagnation level, $\bar{h}_{B}^{*}=\bar{h}_{B}$ $+\left(\pi / \widehat{h}_{B}\right)^{2}$, has a minimum coinciding exactly with the situation in which $F_{0}$ is equal to unity. Differentiating $\bar{h}_{B}^{*}$ with respect to $\bar{h}_{B}$ gives

$$
\frac{\partial \bar{h}_{B}^{*}}{\partial \bar{h}_{B}}=1-\frac{2 \pi^{2}}{\bar{h}_{B}^{3}}
$$

which is zero when $\bar{h}_{B}=\left(2 \pi^{2}\right)^{1 / 3}=K_{c r}$.

Another point of interest is revealed by the plot of cusp depth to source depth $\bar{h}_{c} / \bar{h}_{s}$ (see Figure 11). Over the entire range of values of $F_{0}$, the depth of the cusp remains between $70 \%$ and $80 \%$ of the source depth. This percentage is consistent with the results obtained for the sloping-base problem.

Finally, we note the behavior of $F$ for changing $F_{0}$. As $F_{0}$ tends toward unity from above, $F$ increases up to 2.21 , and this trend appears to continue into the region where $F_{0}<1$. The value of $F$ at $F_{0}=0$ is only 1.776 , however, which is less than $F$ at $F_{0}=1$, which means this trend must reverse in the region $0<F_{0}<1$ if steady solutions exist.

\section{Conclusions}

The amount of material in the literature with which we can compare our results is limited. Craya [2] founded almost all of his relevant work on the exact solution in Section 2, and his assumption that changes in boundary shape would cause little or no change in flow behaviour. His predictions of $F$ for the sloping base problem are all less than about $15 \%$ different to those values calculated in the present paper, but the usefulness of the assumption is doubtful for other flow configurations, such as those in Section 4.

Gariél [3] performed experiments at $F_{0} \doteq 0.1$ to compare with Craya's prediction of $F$ at $F_{0}=0$, i.e. for the source in an infinitely "deep" fluid. His result that $F=1.52$, is very close to Craya's estimate of $F=1.51$. However, the value calculated to high accuracy in $I$ is $F=1.78$. Unfortunately, $F_{0}=0.1$ lies in the region where we were unable to obtain results, making comparison difficult.

Huber [5] used a relaxation method to calculate a series of the two fluid type flows discussed in the introduction. When he took the limit of the Froude number in the lower fluid as the upper fluid slowed, he obtained a value of $F=1.66$ for the flow corresponding to the situation with a source on a solid, horizontal plane, beneath the free surface i.e. to $\gamma=\frac{1}{2}$ in Section 3. The value obtained in the present work was $F=1.44$. It is interesting to note that the assumption of Craya gives a value of $F$ approximately half of these two values.

Note that the results of both Huber and Gariél give values of the Froude number based on source depth, $F$, within the range of all solutions with nonzero flow at infinity calculated in the present work i.e. $1.44 \leqslant F \leqslant 2.16$. 
The method used in this paper and in $I$, appears to give very satisfactory results for those flows which have a cusp, and no extraordinary behaviour, such as waves, on the free surface. The transformations (13) and (20) for $z(t)$ are not unique, however, and better choices, which build in more of the flow behaviour, almost certainly exist.

Finally, the results obtained do not seem to shed any light on why the cusp-like flows change as they do for changes in the geometrical configuration of the solid boundaries. A clue to the reasons may be given by the form of the sub-critical flows, and work is continuing on this problem.

\section{References}

[1] M. Abramowitz and I. A. Stegun (eds.), Handbook of mathematical functions (Dover, New York, 1970).

[2] A. Craya, "Theoretical research on the flow of nonhomogeneous fluids", Houille Blanche 4 (1949), 44-55.

[3] P. Garièl, "Experimental research on the flow of nonhomogeneous fluids," Houille Blanche 4 (1949), 56-64.

[4] C. Domb and M. F. Sykes, "On the susceptibility of a ferromagnetic above the Curie point", Proc. Roy. Soc. London Ser. A 240 (1957), 214-228.

[5] D. G. Huber, "Irrotational motion of two fluid strata toward a line sink" J. Engng. Mech. Div. Proc. Amer. Soc. Civ. Engrs. 86, EM4 (1960), 71-85.

[6] D. H. Peregrine, "A line source beneath a free surface", Mathematics Research Center, Univ. Wisconsin Rep. 1248 (1972).

[7] C. Sautreaux, "Mouvement d'un liquide parfait soumis à la pesanteur. Détermination des lignes de courant", J. Math. Pures Appl. (5) 7 (1901), 125-159.

[8] E. O. Tuck and J. M. Vanden Broeck, "A cusp-like free-surface flow due to a submerged source or sink", J. A ustral. Math. Soc. Ser B 25 (1984), 443-450.

[9] E. O. Tuck, "On air flow over free surfaces of stationary water", J. Austral. Math. Soc. Ser. B 19 (1975), 66-80.

[10] J. M. Vanden Broeck, L. W. Schwartz and E. O. Tuck, "Divergent low-Froude-number series expansion of non-linear free-surface flow problems", Proc. Roy. Soc. London Ser A 361 (1978), 207-224.

[11] M. Van Dyke, "Analysis and improvement of perturbation series", Quart J. Mech. Appl. Math. 27 (1974), 423-450.

[12] J. V. Wehausen and E. V. Laitone, "Surface waves", in Handbuch der Physik 9 (ed. S. Flügge), (Springer, Berlin, 1960).

[13] C. S. Yih, Dynamics of non-homogeneous fluids (Macmillan, New York, 19675). 